ARTIFICIAL CELLS, BLOOD SUBSTITUTES, AND BIOTECHNOLOGY

Vol. 31, No. 3, pp. 303-312, 2003

\title{
Encapsulating Protein into Preformed Liposomes by Ethanol-Destabilized Method
}

\author{
Ching-Hua Wang and Yi-You Huang, Ph.D.* \\ Institute of Biomedical Engineering, College of Engineering, College \\ of Medicine, National Taiwan University, Taipei, Taiwan
}

\begin{abstract}
This study describes a highly efficient method for encapsulating protein drugs into liposomes without using toxic solvents such as chloroform. Large unilamellar vesicles (LUVs) were formed by the ethanol injection method. The effects of composition of phospholipid, buffer concentration, incubation time, incubation temperature, drug loading, ethanol content, and the presence of poly(ethylene) glycol (PEG) lipids on the entrapment efficiency of protein were investigated. It was shown that these preformed LUVs could be induced to entrap protein drugs in the presence of ethanol. Protein could be efficiently encapsulated into liposomes. The interaction of the liposomes with proteins leads to the
\end{abstract}

*Correspondence: Yi-You Huang, Ph.D., Institute of Biomedical Engineering, College of Engineering, College of Medicine, National Taiwan University, No.1, Sec.1, Jen-Ai Road, Taipei, Taiwan; Fax: 886-2-23940049; E-mail: yyhuang@ ha.mc.ntu.edu.tw.

303

DOI: $10.1081 / \mathrm{BIO}-120023160$

1073-1199 (Print); 1532-4184 (Online)

Copyright (C) 2003 by Marcel Dekker, Inc.

www.dekker.com 
formation of multilamellar liposomes ranging in size from 70 to $120 \mathrm{~nm}$, only slightly bigger than the parent LUVs from which they originated. Protein drugs were stable in the liposomal solution. There is no significant activity loss during the encapsulation process. The optimal encapsulation efficiency was achieved when $30 \% \sim 40 \%$ ethanol was used in encapsulating protein drugs. Due to the steric hindrance, LUVs containing a PEG coating will dramatically reduce the encapsulation efficiency, even in liposomes containing very low amount of PEG.

Key Words: Liposomes; Protein drugs; Encapsulation.

\section{INTRODUCTION}

Management of illness through medication has recently entered a new era in which a growing number of peptide-/protein-based therapeutic agents have been produced by biotechnology processes and approved by FDA for therapeutic uses. The efficient and safe delivery of therapeutic proteins is the key to commercial success. Peptides and proteins, such as insulin and vasopressin, are usually administered by injection because, when taken orally, they are degraded by the proteolytic enzymes in the gastrointestinal tract, or they cannot cross the intestinal mucosa because of their hydrophilicity and large molecular size. Most protein products are delivered by parenteral routes such as intravenous (i.v.), intramuscular (i.m.), or subcutaneous (s.c.). Due to the short half-lives of peptide/protein drugs, frequent injections are required. This is an obvious disadvantage, since the drug generally would have to be administered by a trained health care professional. This mode of delivery is always associated with some risk (Huang and $\mathrm{Wu}, 1996)$. Though emerging protein delivery methods have been developed (Cleland and Daugherty, 2001), there are many obstacles remaining to protein/peptide drug delivery by noninvasive routes, for example low absorption and rapid metabolism at the site.

In order to avoiding the rapid degradation and metabolism, and providing sustained release of bioactive proteins and peptides, new formulations needed to be developed. Formulation of proteins and peptides often is more challenging than formulation of small molecules because of the important role of protein conformation as well as the potential for chemical degradation (Clark and Shire, 2000). Liposomes have shown great potential as a drug delivery system. An assortment of molecules, including peptides and proteins, have been incorporated in liposomes, which can then be administered by different routes. Various amphiphatic lipids have been used to formed the liposomes, and the method of preparation can be tailored to control their size 
and morphology (Weiner et al., 1989). Drug molecules can either be encapsulated ion the aqueous space or intercalated into the lipid bilayer.

Incorporation of liposomes into protein formulations for drug delivery systems may represent an attractive method for sustained release, reducing the degradation and enhancing the pulmonary permeation rate (Saari et al., 1999). The engineering of an effective liposomal drug formulation for drug delivery must take into consideration encapsulation efficiency, vesicle stability, and the leakage problem associated with the delivery process. Use of drug-containing liposomes in therapeutics requires techniques of effective drug loading in vesicles that retain their stability in the biological milieu and exhibit optimal biodistribution profiles (Gregoriadis, 1995).

Typical methods used for encapsulating peptides/proteins in lipid vesicles result in very low encapsulation efficiencies. Semple et al. (2001) described a novel formulation process that utilizes an ionizable aminolipid (1,2-dioleoyl3-dimethylammonium propane, DODAP) and an ethanol-containing buffer system for encapsulating large quantities $(0.15-0.25 \mathrm{~g}$ ODN/g lipid) of polyanionic ODN in lipid vesicles. They also added poly(ethylene glycol)lipid during the formulation process to prevent aggregation and intended for intravenous use. Maurer et al. (2001) used preformed vesicles and ethanol destabilized method to encapsulate polynucleotides. They also investigated the effect of ethanol and the presence of poly(ethylene) glycol (PEG) lipids on the interaction of nucleotide-based polyelectrolytes with cationic liposomes. Zadi and Gregoriadis (2000) reported a high-yield entrapment method for encapsulating solutes into small liposomes by dehydration and rehydration. In this study, we developed a highly efficient method for encapsulating protein/ peptide drugs into liposomes without using toxic solvents such as chloroform. The drug was entrapped into liposomes by preformed vesicles and ethanoldestabilized method. The relationship between encapsulation efficiency and composition of phospholipid, buffer concentration, incubation time, incubation temperature, and drug loading were investigated.

\section{MATERIALS AND METHODS}

\section{Materials}

Hydrogenated egg yolk PC (Type I-EH, highly purified egg lecithin hydrogenated in the presence of a platinum catalyst, 99\% phosphatidylcholine), Egg yolk PC (Type X-E, 60\% phosphatidylcholine), cholesterol (Chol), Insulin, bovine serum albumin and phosphate buffered saline were purchased from Sigma Chemical Company (St. Louis, MO, USA). PEG-DPPE (Nmethoxypolyethyleneglycol) succinoyl-2-N-dipalmitoylphosphatidyl ethano- 
lamine, PEG Mw = 5000) was purchased from NOF Corporation (Hyogo, Japan). Chloroform, methanol, n-butanol and other chemicals used in the experiments and analysis were reagent grade (Wako Pure Chemicals, Japan).

\section{Encapsulating Protein Drugs into Liposomes}

Different liposome compositions were evaluated according their physical and chemical stability. Large unilamellar liposomes were prepared by slow addition of the lipids dissolved in ethanol $(0.4 \mathrm{ml})$ to $\mathrm{pH} 4$ citrate buffer $(0.6 \mathrm{ml})$ followed by extrusion through 2 stacked $200 \mathrm{~nm}$ filters (3 passes). The final ethanol concentration was $40 \%[\mathrm{v} / \mathrm{v}]$ and the lipid composition as shown in Table 1. Albumin or insulin solution was slowly added while vortexing. Subsequently the ethanolic dispersion was incubated at $40^{\circ} \mathrm{C}$ for $1 \mathrm{hr}$, dialyzed for $2 \mathrm{hrs}$ against citrate buffer and twice against HBS (20 mM HEPES/145 mM NaCl) to remove excess ethanol. Untrapped drugs were removed by a sepharose CL-4B gel column (Huang et al., 2000; Maurer et al., 2001).

Particle size and size distributions of liposomes were measured by quasielastic light scattering (QELS) and freeze-fracture electron microscopy. QELS measurements employed a Coulter Model N4 Plus sub micro particle analyzer (Hialeah, FL, USA). Sample polydispersity was evaluated by the goodness-of-fit parameter $\chi^{2}$, where values of $\chi^{2}<3$ indicate monodisperse formulations (according to the specification by the manufacture.)

\section{Determination of Encapsulation Efficiencies}

Albumin concentration was determined by BCA assays. Lipid concentrations were determined by Steward assay (by the formation of a

Table 1. The effects of different lipid composition on the protein encapsulation ratio.

\begin{tabular}{lc}
\hline $\begin{array}{l}\text { Lipid composition (mole\%) } \\
\text { (HPC/Chol/DODAP/PEG) }\end{array}$ & $\begin{array}{c}\text { Encapsulation } \\
\text { ratio }(\%)\end{array}$ \\
\hline $70 / 30 / 0 / 0$ & 44.60 \\
$40 / 60 / 0 / 0$ & 32.33 \\
$40 / 40 / 20 / 0$ & 32.86 \\
$40 / 40 / 19 / 1$ & 30.58 \\
$70 / 30 / 0 / 1$ & 36.70 \\
$65 / 30 / 0 / 5$ & 18.90 \\
$30 / 65 / 0 / 5$ & 10.11 \\
\hline
\end{tabular}


complex with ammonium ferrothiocyanate in organic solution) after separation from albumin by a Bligh and Dyer extraction. Encapsulation efficiencies are expressed as protein to lipid ratios [w/w].

\section{RESULTS AND DISCUSSION}

Preformed vesicles were formed by ethanol injection. After extruding by three pass through two stacks of $1 \mu \mathrm{m}$ polycarbonate membranes three passes, the preformed vesicles were with size of $90 \pm 20 \mathrm{~nm}$ (QELS measured) before entrapping the protein. Peptide/protein drugs could be efficiently encapsulated into preformed vesicles by incubating the protein with liposomes containing ethanol, followed by detergent dialyzing procedures to remove the ethanol. The interaction of liposomes with proteins leads to the formation of multilamellar liposomes ranging in size from 70 to $120 \mathrm{~nm}$, only slightly bigger than the parent LUVs from which they originated. Although the morphology of the liposomes is still preserved at the ethanol concentrations used for entrapment $(25-40 \%, \mathrm{v} / \mathrm{v})$, the lipid can flip-flop across the membrane and exchange rapidly between liposomes and entrap the protein into the liposome. After removing the ethanol, protein drugs were stable in the liposomal solution. There is no significant activity loss during the encapsulation process.

The entrapping efficiency depends on many factors, including the ionic strength of citrate buffer, the $\mathrm{pH}$ of citrate buffer, the incubation time, the drug loading ratio, and lipid composition etc. The encapsulation efficiency was as high as $45 \%$ when liposomes were made up of HPC/Chol $(70 / 30 \mathrm{~mol} \%)$, as shown in Table 1. While increasing the proportion of cholesterol to HPC or adding PE-PEG, the encapsulation efficiency decreased. As increasing the cholesterol ratio could strengthen the stability of lipid bilayer, the lipid bilayer became more rigid and the fluidity was reduced causing lower encapsulation efficiency.

Adding DPPE-PEG, which inserted into lipid bilayer and caused threedimensional steric hindrance around the liposomes, would keep drugs from entering the inside of the vesicles and decrease the encapsulation efficiency.

Figure 1 shows the effect of the concentration of ethanol on the trapping efficiency. When the concentration of ethanol was 30\%, the trapping efficiency was highest. Because ethanol is a solvent of lipid and posses the surfactant property, it can make the membrane become more flexible. If without adding ethanol, the bilayer would be too rigid to encapsulate drugs. However, if the concentration of ethanol was too high ( $>30 \%$ ), the encapsulation efficiency became lower because the high fluidity of the bilayer would increase loss of encapsulated drug and 


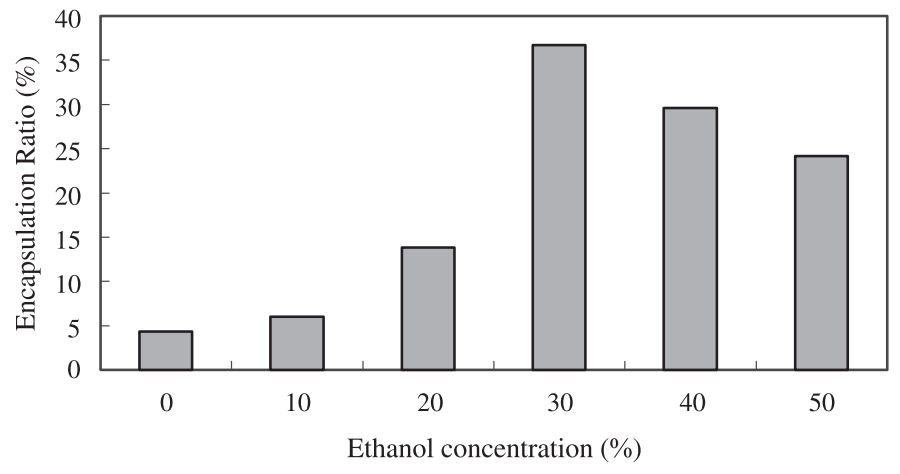

Figure 1. The effect of ethanol concentration on the protein encapsulation ratio.

decrease the trapping efficiency. Figure 2 shows the effect of incubation temperature on the trapping efficiency. When the incubation temperature was increased to $40^{\circ} \mathrm{C}$, the trapping efficiency would be better. At higher temperatures, the structure of the lipid membrane could become more irregular and looser so as to raise the permeability of the lipid bilayer. Drugs would pass through the bilayer easily and, as a result, the trapping efficiency was higher. Figure 3 shows the effect of incubation time on the trapping efficiency. The longer the incubation time, the higher the trapping efficiency. But if the incubation time was too long ( $>3 \mathrm{hrs}$ ), the amount of encapsulated drugs would reach saturation and the trapping efficiency didn't become higher.

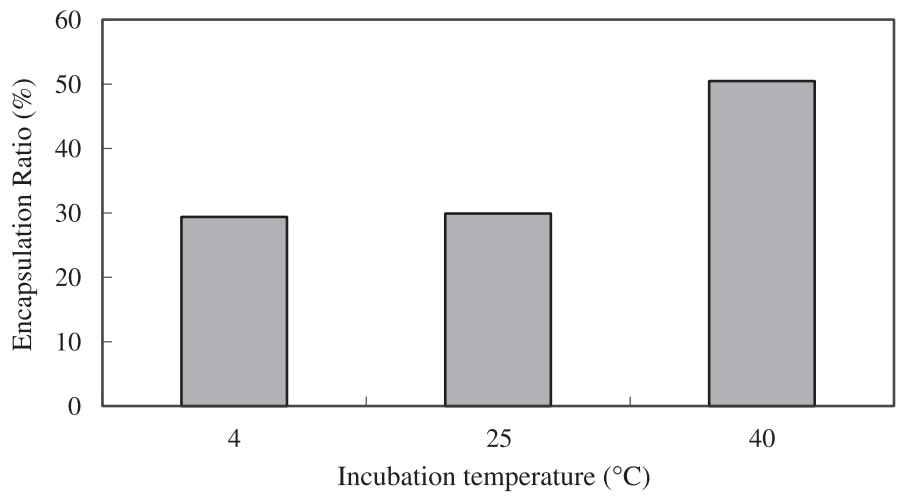

Figure 2. The effect of incubation temperature on the protein encapsulation ratio. 


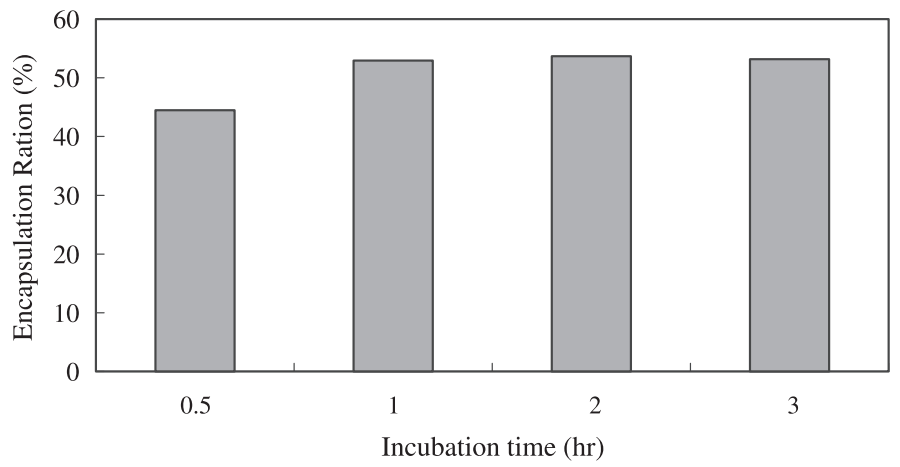

Figure 3. The effect of incubation time on the protein encapsulation ratio.

The optimal incubation time was 1 to 2 hours. Figure 4 shows the effect of buffer concentration on the trapping efficiency. For HPC/Chol/PEPEG (70/30/1mol\%) composition system, at lower citrate concentration, the trapping ratio was low, while increasing the citrate concentration would increase the protein-trapped ratio. Due to the competing effects, if the citrate concentration was more than three-fold higher than drug concentration, the trapping efficiency dropped dramatically. These results suggested that there is a maximal condition. When the initial albumin/(total lipid amount) loading ratio $=0.2 \mathrm{mg}$ protein $/ \mathrm{mg}$ of lipid, at citrate buffer concentration of $100 \mathrm{mM}$, we can get the highest encapsulation efficiency, approximately $52 \%$. This point seems to be changed if we change the

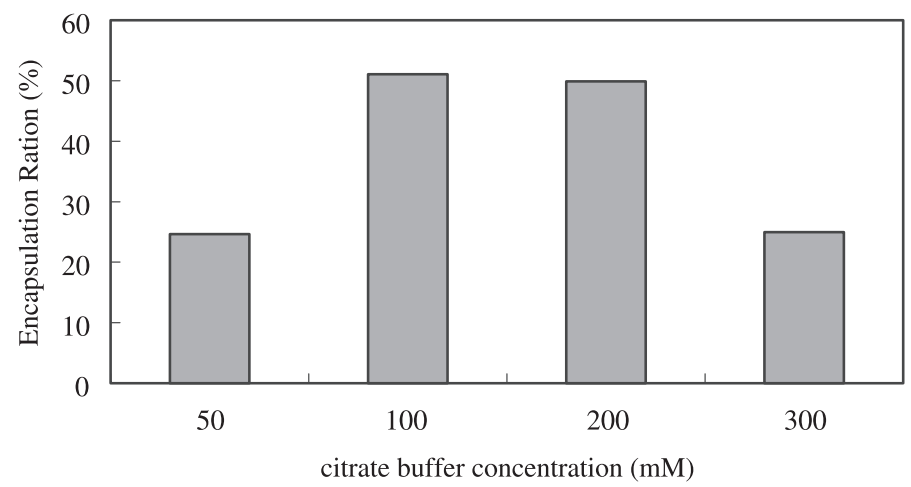

Figure 4. The effect of citrate buffer concentration on the protein encapsulation ratio. 


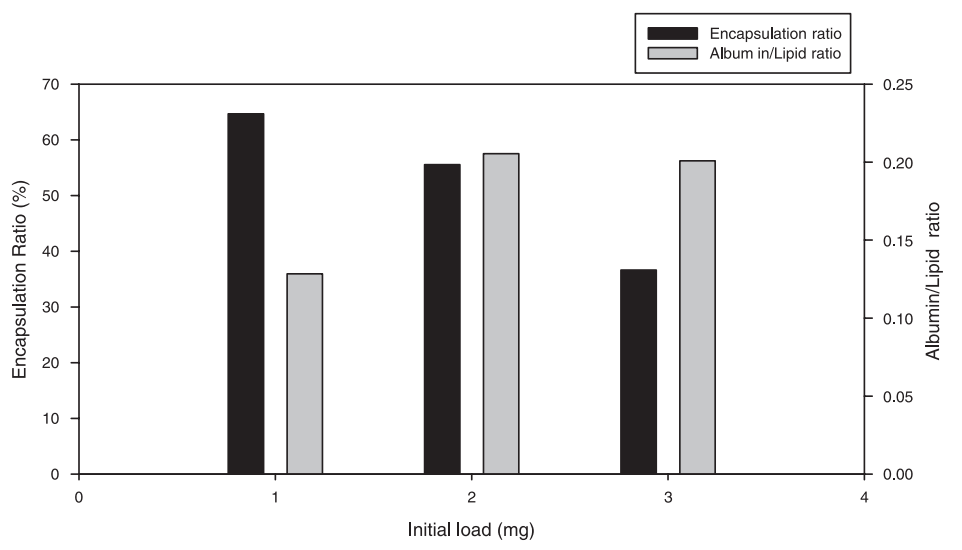

Figure 5. The effect of drug loading on the protein encapsulation ratio.

protein loading amount. The vesicle size increases slightly with increasing ionic strength. The effect of drug loading amount on the trapping efficiency is shown in Figure 5. The encapsulated drug amount increased as drug loading amount increased, while the trapping efficiency decreased. Because the drug-containing capacity of the vesicle is limited, if drug loading amount was too high, it would cause a waste of drug and lower the

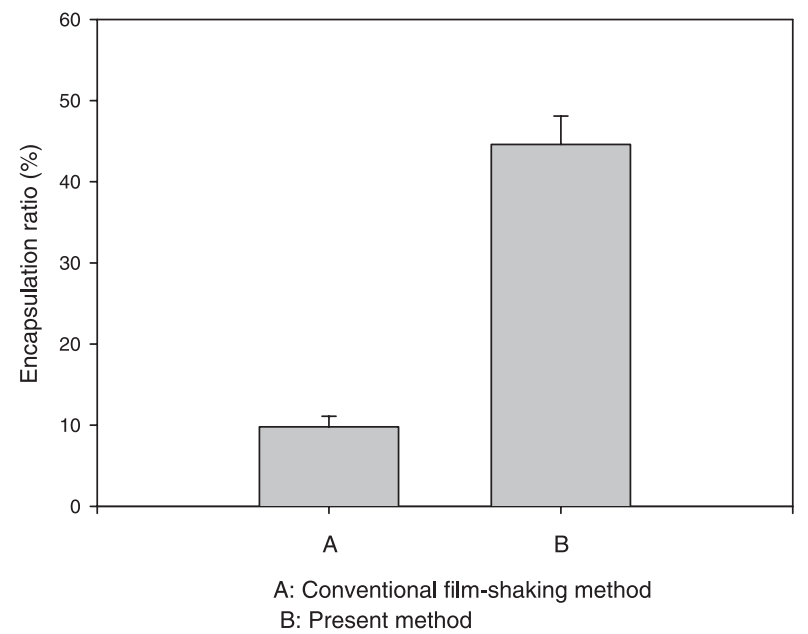

Figure 6. Comparison of encapsulation ratio between present method and conventional film shaking method. 
trapping efficiency rather than increase the encapsulated drug amount. Comparing to the conventional film shaking method, dripping ethanol method and detergent-dialyzing procedures could achieve $4 \sim 5$-fold higher trapping efficiency, as shown in Figure 6. Using the new method could have enough incubation time and high reaction temperature to make more drug encapsulated into liposome so the trapping efficiency would be higher.

\section{CONCLUSIONS}

Peptide/protein drugs could be efficiently encapsulated into liposomal carriers. The liposomal carrier provides sustained release for the peptide and protection from degradation during drug delivery. The optimal encapsulation efficiency was achieved when $30 \%$ ethanol was used in encapsulating protein drugs. The high entrapment efficiencies, small size, and neutral character of these novel liposomal systems are of utility for liposomal delivery of macromolecular drugs. Due to the steric hindrance, LUVs containing a PEG coating will reduce the encapsulation efficiency dramatically, even using a very low amount of PEG.

\section{ACKNOWLEDGMENT}

This study was supported by National Science Council, Taiwan NSC89-2320-B-002-143-M08.

\section{REFERENCES}

Clark, A. R., Shire, S. J. (2000). Formulation of proteins for pulmonary delivery. In: McNally, E. J., ed. Protein formulation and delivery. New York: M. Dekker. pp. 201-235.

Cleland, J. L., Daugherty, A., Mrsny, R. (2001). Emerging protein delivery methods. Curr. Opin. Biotechnol. 12:212-219.

Gregoriadis, G. (1995). Engineering targeted liposomes: progress and problems. Trends Biotechnol. 13:527-537.

Huang, Y. Y., Wu, S. H. (1996). Transdermal iontophoretic delivery of thyrotropin-release hormone across excised rabbit pinna skin. Drug Dev. Ind. Pharm. 22:1075-1081.

Huang, Y. Y., Maurer, N., Cullis, P. R. (2000). Developing serum-stabilized cationic liposome-DNA particles for gene therapy by preformed 
vesicles and ethanol method. Biomed. Eng.: Appln. Basis Commu. 12:217-222.

Maurer, N., Wong, K. F., Stark, H., Louie, L., McIntosh, D., Wong, T., Scherrer, P., Semple, S. C., Cullis, P. R. (2001). Spontaneous entrapment of polynucleotides upon electrostatic interaction with ethanoldestabilized cationic liposomes. Biophys. J. 80:2310-2326.

Saari, M., Vidgren, M. T., Koskinen, M. O., Turjanmaa, V. M., Nieminen, M. M. (1999). Pulmonary distribution and clearance of two beclomethasone liposome formulations in healthy volunteers. Int. J. Pharm. 181:1-9.

Semple, S. C., Klimuk, S. K., Harasym, T. O., Dos Santos, N., Ansell, S. M., Wong, K. F., Maurer, N., Stark, H., Cullis, P. R., Hope, M. J., Scherrer, P. (2001). Efficient encapsulation of antisense oligonucleotides in lipid vesicles using ionizable aminolipids: formation of novel small multilamellar vesicle structures. Biochim. Biophys. Acta 1510(1-2):152166.

Weiner, N., Martin, F., Riaz, M. (1989). Liposomes as a drug delivery system. Drug Dev. Ind. Pharm. 15:1523-1554.

Zadi, B., Gregoriadis, G. (2000). A novel method for high-yield entrapment of solutes into small liposomes. J. Liposome Res. 10:73-80. 
Copyright $\odot 2003$ EBSCO Publishing 